\title{
Accuracy in mineral identification: image spectral and spatial resolutions and mineral spectral properties
}

\author{
Maria Sgavetti, Ilaria Longhi, Sandro Meli and Loredana Pompilio \\ Dipartimento di Scienze della Terra, Università degli Studi di Parma, Italy
}

\begin{abstract}
Abstact
Problems related to airborne hyperspectral image data are reviewed and the requirements for data analysis applied to mineralogical (rocks and soils) interpretation are discussed. The variability of mineral spectral features, including absorption position, shape and depth is considered and interpreted as due to chemical composition, grain size effects and mineral association. It is also shown how this variability can be related to well defined geologic processes. The influence of sensor noise and diffuse atmospheric radiance in classification accuracy is also analyzed.
\end{abstract}

Key words reflectance spectroscopy - VIS-SWIR rock identification - spectral resolution - spatial resolution - noise effects

\section{Introduction}

\subsection{Premise}

Airborne spectroscopy perhaps needs to rediscover one of its most important original aspects, and be regarded again as a scientific research tool whose purpose is the measurement of physical variables, the study of phenomena and processes, the definition of calibration and data analysis procedures, as well as the simulation of specific characteristics for future space borne systems. This perspective does not imply a merely academic or philosophical exercise, disregarding practical problems. On the contrary, it provides the very answer to the need for establishing well grounded conceptual frame-

Mailing address: Dr. Maria Sgavetti, Dipartimento di Scienze della Terra, V.le G.P. Usberti 157/A, Campus Universitario, 43100 Parma, Italy; e-mail: maria.sgavetti@ipruniv.cce.unipr.it works for any kind of remote sensing applications requiring high resolution spectral data, and it also provides the fundamental requirements that will lead the new technology for the next generations of satellite sensors.

\subsection{From qualitative to quantitative}

Remote sensing image spectroscopy is a tool for the measurement of physical quantities. It represents an application, at various spectral and spatial resolutions, of reflectance and emittance laboratory spectroscopy. As such, laboratory spectroscopy has accompanied remote sensing data analysis from the outset, to aid interpretation of the spectral properties of rocks, soils and sediments. Even though the low spectral resolution of multispectral remote sensors did not allow the exploitation of the spectral variability of geologic materials, it was sometimes possible to derive «second order» criteria, making it possible to discriminate groups of rocks beyond the spectral resolution of the data. One example, more extensively discussed later in this paper, was the recognition of rocks with a different metamorphic grade, based on the wavelength shift, in the order of ten to fifteen nanometers, of 
the $2200 \mathrm{~nm}$ Al-OH vibrational band. First proposed by Duke (1994), the relationship between the Al-OH position and the metamorphic grade was further discussed by Longhi et al. (2000), including considerations relative to the rock bulk composition. Moreover, it was observed that the absorption peak wavelength shift in quartzites was also associated with an increase in band intensity, both variations being due to the same compositional variation in the rocks. As a result, the more intense absorption at wavelengths comprised within the TM band 7 allowed the discrimination between low and high-grade metamorphic rocks, even with low-resolution data (Longhi et al., 2000, 2001).

The advent of airborne high-resolution spectrometers provided new possibilities for mineral identification and quantitative determination. At the same time, it enhanced the importance of specific problems, related to physical, mineralogical, geochemical variables and processes.

In this paper, we present an overview of the problems commonly encountered in short wave infrared (SWIR) remote sensing spectroscopy for determining the mineralogical composition of a surface, which is at the basis of any geological and geo-environmental study. The most important problems in image classification include 1) the pixel heterogeneity and spectral variability inherent to many rocks and soils, both determining the choice of the sensor spectral and spatial resolutions, and 2) the image data quality to which the sensor signal to noise ratio makes a primary contribution.
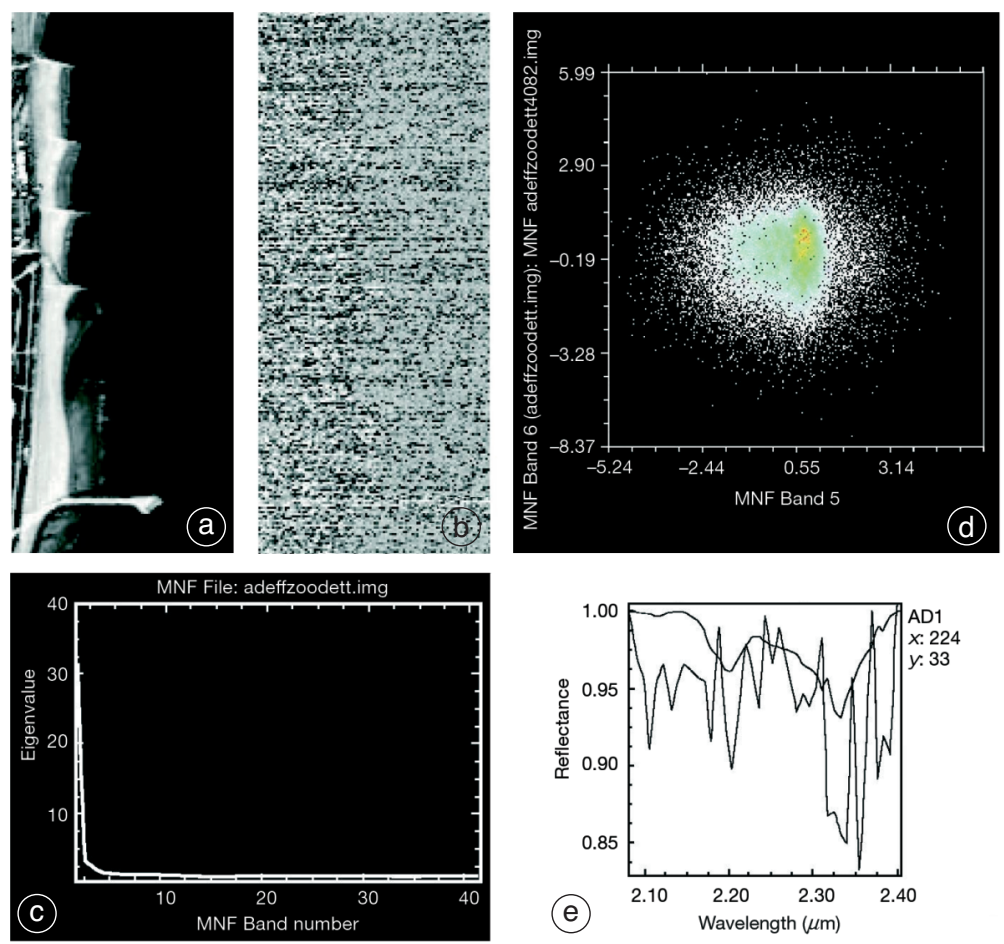

Fig. 1a-e. a) Evaluation of the inherent dimensionality in the data for a MIVIS image of a sandy littoral area. MNF transformation was applied to the 2-2.5 mm interval, where MIVIS sensor has spectral resolution adequate for mineralogical analysis: b) MNF band 5, c) eigenvalue plot, and d) MNF band 5 and 6 scatterplot document high noise content in the data; e) shows a comparison between one endmember and the MIVIS spectrum of a pixel classified through SAM (cf. fig. 2a,b); spectral contrast is in the order of 0.05 . 

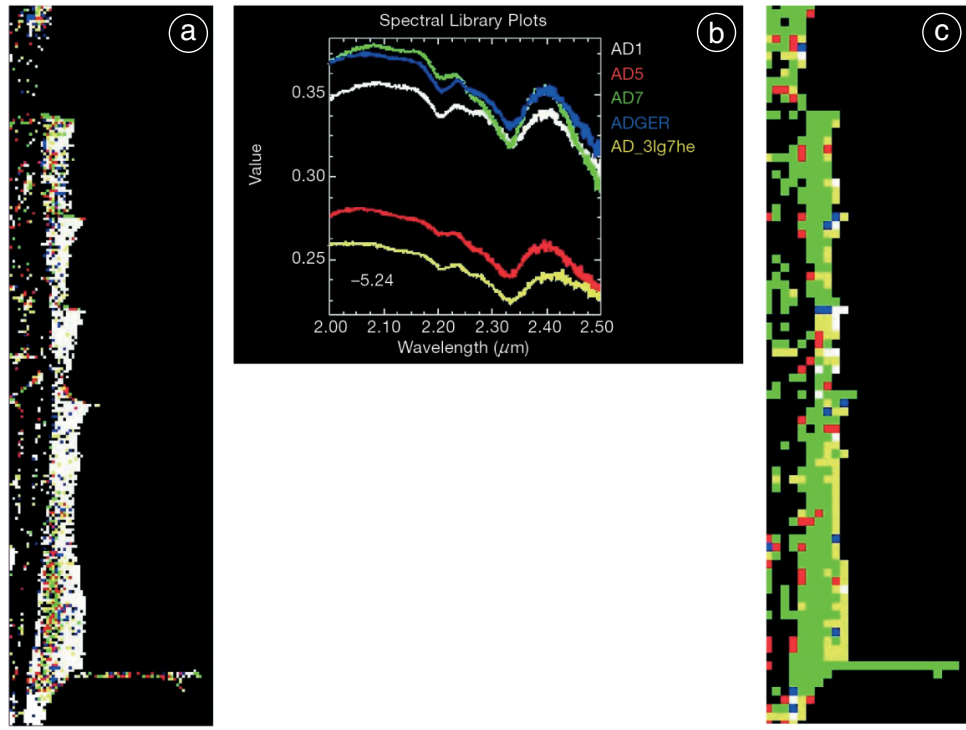

Fig. 2a-c. a) SAM classification of the image of fig.1a, with $3 \mathrm{~m}$ ground resolution; b) endmember library: white, green and blue: $>90 \%$ of light mineral fraction; composition: quartz, dolomite, calcite, feldspars, micas; the spectrum coded white has the lowest carbonate content; red and yellow: about $70 \%$ of heavy mineral fraction; composition: quartz, dolomite, calcite, hornblende, clinopyroxene, muscovite, biotite, feldspars; c) SAM classification of the same image, with a degraded ground resolution of $9 \mathrm{~m}$.

\section{Image classification and sensor spatial resolution}

Generally, the spectrum of a heterogeneous pixel is assumed to result from a linear combination on a macroscopic scale of the spectra of the materials occupying distinct areas within the pixel (Singer and McCord, 1979). The classification of multicomponent pixels is based on unmixing techniques, assuming that the degree of the match between unknown and reference spectra is indicative of the apparent fractional abundance of the component materials (Boardman, 1993). As such, these techniques are particularly sensitive to the noise level in the data requiring an accurate evaluation of the inherent dimensionality of the image data. Figure 1a-e shows the results of the evaluation of $2-2.5 \mu \mathrm{m}$ (SWIR) Multispectral Infrared and Visible Imaging Spectrometer (MIVIS) data, with sensor mean signal to noise ratio of 130 (after pre-flight calibration) in the 2-2.5 $\mu \mathrm{m}$ region (fig. 1a), where important diagnostic absorption bands of several minerals are present. Noise dominated Minimum Noise Fraction (MNF) band 5 (fig. 1b), eigenvalue plot (fig. 1c) and MNFs 5 and 6 scatterplot (fig. 1d) give evidence that these data are not suitable for mineral quantitative abundance determination through unmixing methods for surfaces with low spectral contrast (fig. 1e).

A qualitative classification procedure is shown in fig. 2a,b, using ancillary field data. Reflectance laboratory spectra were measured on samples of the endmember materials recognized in the area (sand samples with different mineralogical composition, mollusk shells, and algae). Linear combinations of endmember spectra were calculated, to obtain mixed spectra simulating pixels with different area percentages of the endmembers. Both original endmembers and calculated spectra were used for a Spectral Angle Mapper classification (SAM) of MIVIS data with a $3 \mathrm{~m}$ ground resolution. The colored pixels densely assembled in the inner part of the beach (fig. 2a) indicate a concentration of sands with a high content of heavy minerals, as well as sand 
mixed with winter vegetation in correspondence of dune deposits. White pixels rather homogeneously distributed in the largest portion of the beach represent high reflectance, quartz rich sands (Crotti et al., 2002).

SAM classification of data with simulated 9 $m$ ground resolution, using the same end-member library as above, would result in a significantly different classification of the beach components both for pixel assignment and for spatial distribution (fig. 2c).

\section{Mineral identification and spectral resolution}

Mineral identification by means of hyperspectral data analysis is based on the recognition of absorption band associations, diagnostic of mineral species. However, a mineral is not univocally determined by a general crystal chemical formula, inasmuch as many ionic substitutions in the crystal lattice occur, leading to continuous variations in the lattice physical properties. On the other hand, spectroscopy is highly sensitive to these variations. As such, it is particularly useful for the recognition of the geochemical processes responsible for the mineral composition changes and, integrated with data of different nature, for the interpretation of the geologic environment in which the rock or sediment formed (Longhi et al., 2000, 2004). A shift in the absorption peak wavelength was observed, for example, in muscovite. The Al-OH vibrational occurs at shorter wavelength when the $\mathrm{Al}^{\mathrm{VI}}$ content increases, and this composition variation can be due to geologic processes such as metamorphism (fig. 3a-d; Longhi et al., 2000), or hydrothermal alteration (Swayze et al., 1992).
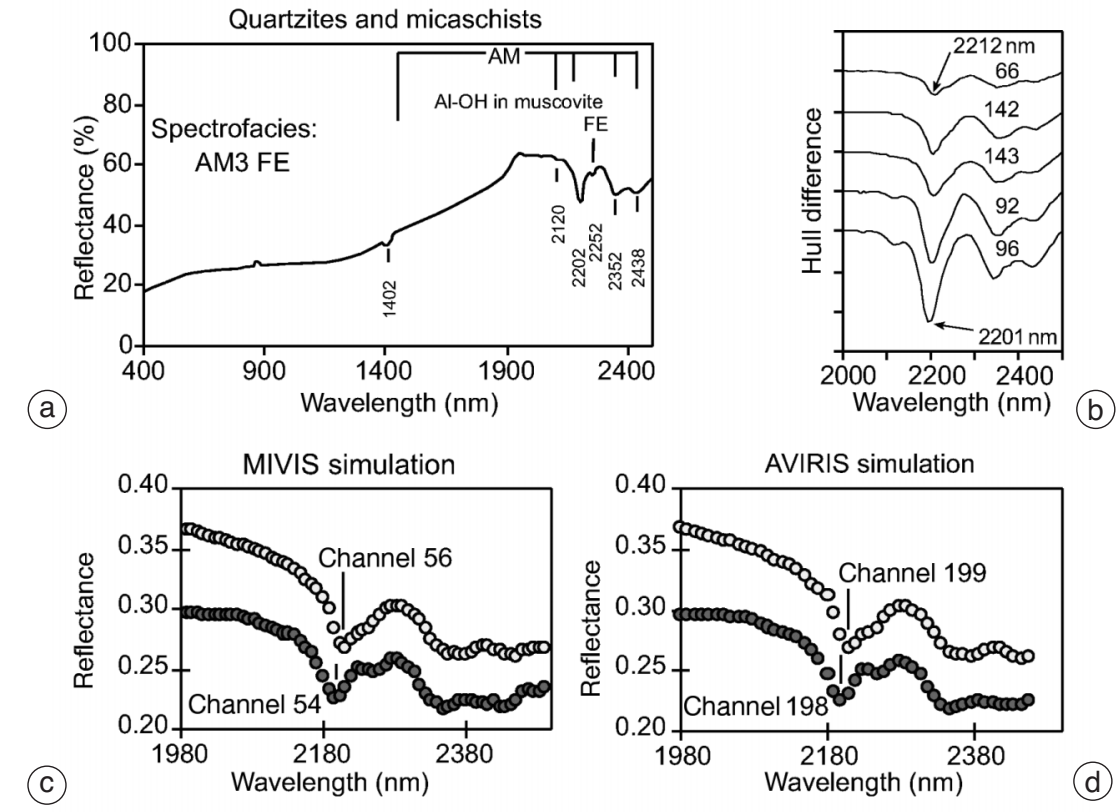

Fig. 3a-d. a) Reflectance spectrum of a quartzite, showing the muscovite absorption band association; AM: Al$\mathrm{OH}$ vibrational; FE: Fe-OH vibrational; b) detail of the 2000-2500 nm interval, showing the wavelength shift of the Al-OH absorption peak toward shorter values, associated with band depth increase; both variations are related to increasing metamorphic grade of the rock; $y$ axis: continuum removed reflectance spectra displaced for clarity, $10 \%$ increment; c) and d) simulation of the spectral response of two hyperspectral sensors, both discriminating the absorption peak shift. 

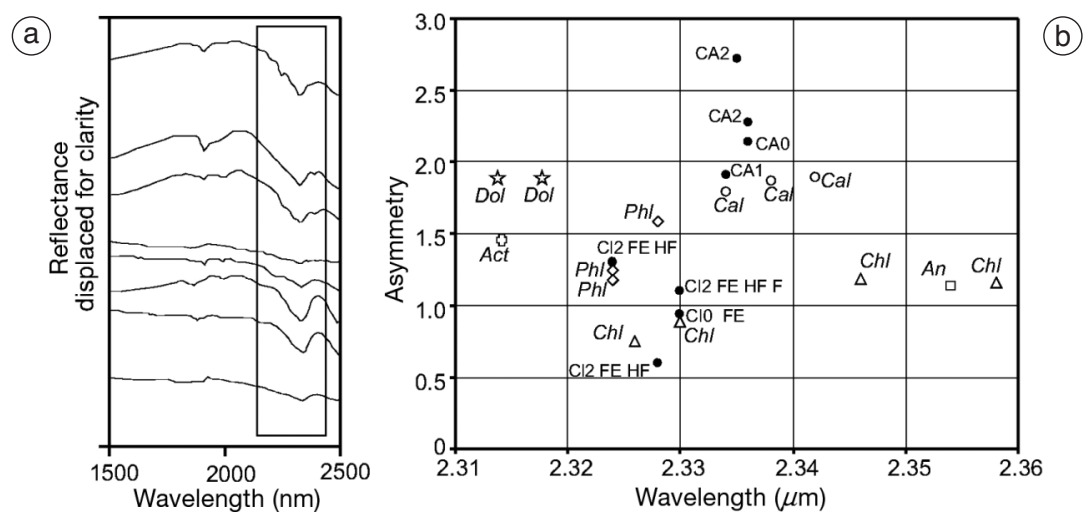

Fig. 4a,b. a) Reflectance spectra of an impure marbles suite, showing the asymmetry variation of the $\mathrm{CO}_{3}{ }^{-2}$ band (in the box); b) $\mathrm{CO}_{3}{ }^{-2}$ band wavelength variation due to marble chemistry variation from calcite (CA) to dolomite (CI), and asymmetry variation due to accessory minerals (see text for discussion); bold circles: marble samples with spectrofacies, i.e. associations of absorption bands due to the sample component minerals (FE: Fe$\mathrm{OH}$ vibrational; $\mathrm{HF}: \mathrm{H}_{2} \mathrm{O}$ and $\mathrm{OH}^{-}$vibrationals in feldspars); open symbols: accessory minerals.

These considerations apply to the particular case of monomineralic rocks or sediments, or, at least, those containing only one spectroscopically active mineral. In a mineral association or mixture in which more than one spectroscopically active mineral is present, the identification of the different mineralogical phases is further complicated when different minerals have partly overlapping absorption bands. Even neglecting the grain size effects, the result is a composite band representing a non-linear combination of the original bands. One example is represented by the shape variation of the carbonate absorption band in a suite of marbles, superimposed on the overall wavelength variation due to chemistry variation (fig. $4 a, b$ ). The presence of accessory minerals such as phlogopite $(\mathrm{Phl})$ increases the left-hand asymmetry of the $\mathrm{CO}_{3}^{-2}$ band in calcitic marbles $(\mathrm{CA})$, whereas the presence of chlorite (Chl) and anorthite (An) reduces the asymmetry in calcitic-dolomitic (CI) marbles (Longhi et al., 2001).

\section{Mineral abundance quantitative determination}

In SWIR spectroscopic analysis, the absorption peak wavelength is related to the energy of a transition between vibrational energy levels, whereas the intensity of a given absorption band can be indicative, with suitable calibration, of the absorbing species concentration (McMillan and Hofmaister, 1988). A quantitative analysis is however possible solely in the case of adequate particle separation, where the radiation is prevented from going through adjacent grains in its path back to the spectrometer. When the grains are close to each other, both the interference among absorption features pertaining to different minerals and the scattering effects combine to give fairly unpredictable results (Nash and Conel, 1974; Singer, 1981; Clark and Roush, 1984; Clark, 1995).

One example is the mixture shown in fig. 5, consisting of several spectrally different minerals, with grain size varying over a wide range including also very small particles in which the band depth is not representative of the mixture composition. The iron about $1300 \mathrm{~nm}$ band dominates the spectrum of a mixture of heavy, iron bearing minerals (about 50\%) and light minerals, overestimating at $70 \%$ the heavy mineral abundance.

This phenomenon represents one of the most critical topics for the remote sensing spectroscopy community, in its attempt to establish 
criteria for the quantitative analysis of mineral mixtures. Indeed, in nature there are cases in which a simple relationship between absorption band intensity and both mineral abundance and grain size can be recognized. In a suite of quartzites, where muscovite is the only spectrally active mineral, the $2200 \mathrm{~nm}$ absorption band of the $\mathrm{Al}-\mathrm{OH}$ vibrational is linearly related to both muscovite abundance and particle size (Longhi

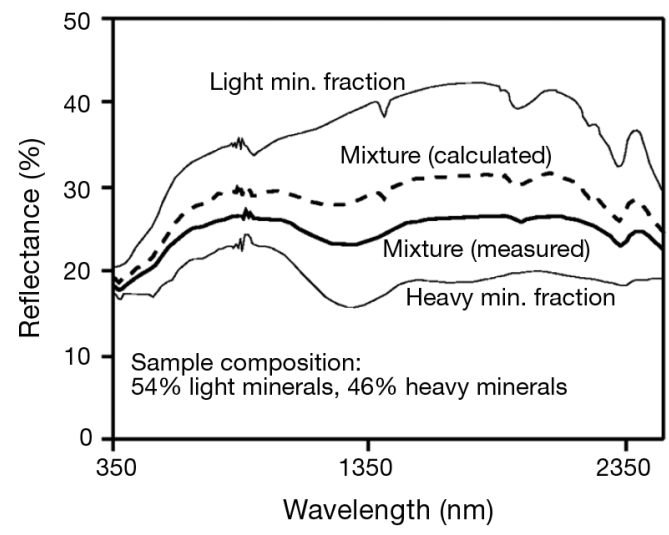

Fig. 5. Measured spectrum of a sand sample from the area of fig. 1a (bold line), relative light and heavy mineral fractions (thin lines), and calculated spectrum (dashed line) with weighted light and heavy fractions corresponding to the original sample composition. The measured sample overestimates the heavy mineral abundance. et al., 2001), according to the Bouguer-BeerLambert Law (fig. 6a,b).

\section{Noise level in the data and spectral feature recognition}

Figure 1a-e shows how the presence of noise in the data might hinder the recognition of relatively weak absorption bands. Noise generating factors include sensor electronics and atmospheric and scene scattering, interacting with sample characteristics. Sensor noise, expressed by the Signal to Noise Ratio (SNR), has a component represented by a normally distributed noise superimposed on the sample spectrum. An algorithm, slightly modified after Oking and Roberts (2000), was used to add noise to the data as a function of wavelength (Sgavetti et al., 2001)

$$
\begin{aligned}
& S_{\text {noisy }}=\left\{\left[\left(S_{\text {lab }} * E_{\text {solar }} * G+O\right) *(1+N(0,1) / \mathrm{SNR})+\right.\right. \\
& -O] / G\} / E_{\text {solar }}
\end{aligned}
$$

where $S_{\text {noisy }}$ is the noisy spectrum, $E_{\text {solar }}$ the solar irradiance, $G$ and $O$ the sensor gain and offset, $N(0,1)$ a random number with a Gaussian distribution with mean equal to zero and standard deviation of 1, and SNR the sensor SNR derived from pre-flight calibration (all parameters being a function of wavelength). Sensor contribution to noise in the data is determined by varying the sensor SNR and assuming a per-
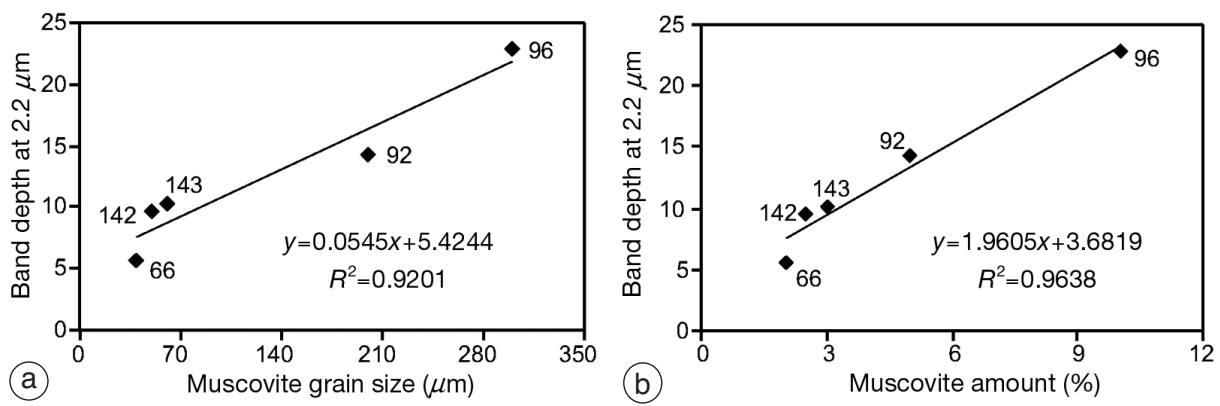

Fig. 6a,b. Relationship between $2200 \mathrm{~nm}$ Al-OH band of muscovite in quartzite, and muscovite grain size (a) and abundance (b). The relationship is linear in these rocks, where muscovite is the only spectroscopically active mineral. 

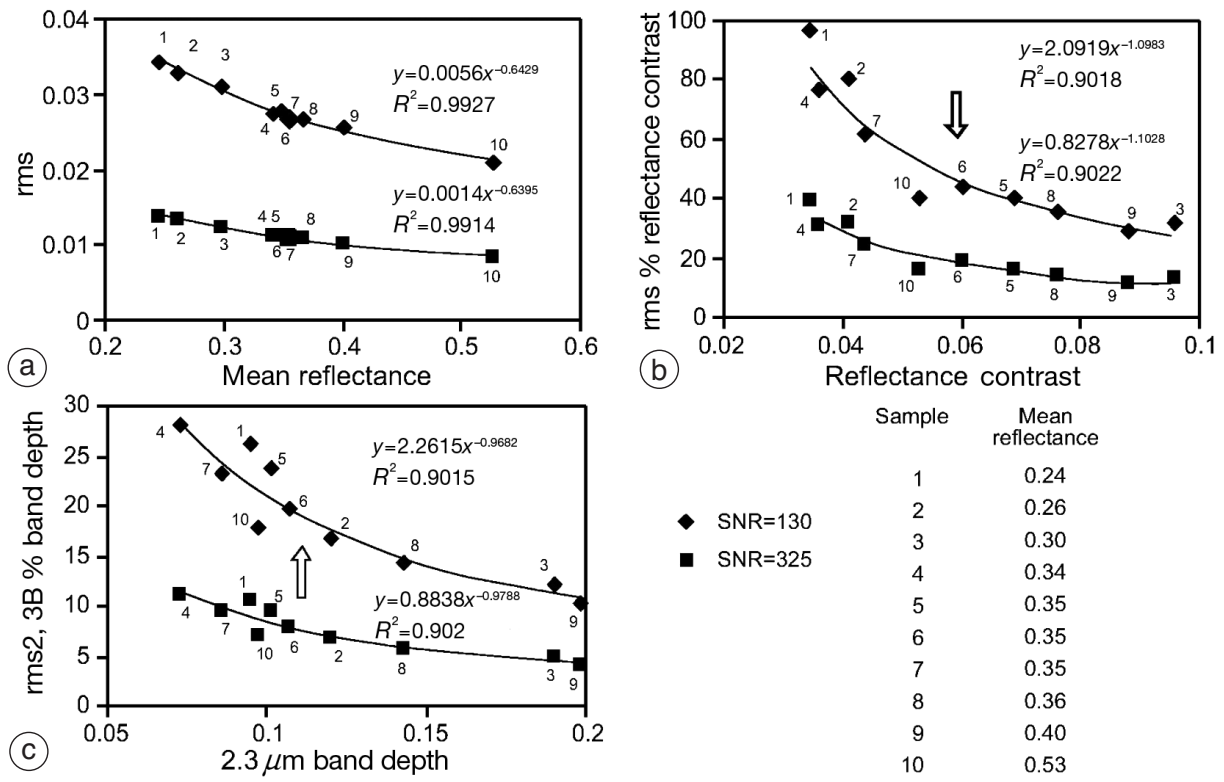

(b)

\begin{tabular}{|c|c|}
\hline Reflectance & contrast \\
\hline Sample & $\begin{array}{c}\text { Mean } \\
\text { reflectance }\end{array}$ \\
\hline 1 & 0.24 \\
\hline 2 & 0.26 \\
\hline 3 & 0.30 \\
\hline 4 & 0.34 \\
\hline 5 & 0.35 \\
\hline 6 & 0.35 \\
\hline 7 & 0.35 \\
\hline 8 & 0.36 \\
\hline 9 & 0.40 \\
\hline 10 & 0.53 \\
\hline
\end{tabular}

Fig. 7a-c. Noise content in the data as a function of sample mean reflectance (a), reflectance contrast (b), and $\mathrm{CO}_{3}{ }^{-2}$ band depth (c), for two sensor SNRs. Noise in the data is expressed as the root mean square of the ratio between noisy and original spectra (rms); in (b) and (c) is expressed as percentage of reflectance contrast and $2.3 \mu \mathrm{m}$ band depth, respectively. Goodness of fit for SNR 130: 0.656; for SNR 350: 0.852. Correct classification for SNR 130: 40\%; for SNR 350: 60\%. Arrows: spectral contrast and band depth threshold values.

fect correction of the atmospheric effects. The noise threshold in the data that can be accepted for mineralogical analysis is determined by analyzing the noisy spectra with reference to sample characteristics, such as the mean reflectance of the spectrum, the spectral reflectance contrast and, for individual absorption bands, the band depth (fig. 7a-c). The data noise reported in the diagrams was calculated for the SWIR region, considering a solar irradiance with a mean of $9.25 \mathrm{~W} * \mathrm{~m}^{-2} * \mu \mathrm{m}^{-1} * \mathrm{sr}^{-1}$, measured at the ground in the area of fig. 1a-e, during a MIVIS flight. Sensor average SNR was equal to 130 (actual sensor SNR) and 325 (simulated). The critical values of sample reflectance contrast and band depth are about 0.06 and 0.11 respectively (arrows in fig. 7b,c). Below these values, the noise is above $50 \%$ of the reflectance contrast for $\mathrm{SNR}=130$ and $20 \%$ for $\mathrm{SNR}=325$, and is above $17 \%$ and $7 \%$ of the band depth for the two SNRs respectively. Using standard classifi- cation methods, such as Spectral Analyst, the average goodness of fit is 0.656 and 0.852 for the two SNRs respectively, and the average probability of correct classification of the noisy spectra is below $40 \%$ for $\mathrm{SNR}=130$ and about $60 \%$ for $\mathrm{SNR}=325$.

Radiometric evaluation includes the increment, due to noise, of output radiance with respect to input radiance for individual samples, and the radiance variation due to atmospheric scattering. In fig. 8, for $\mathrm{SNR}=130$ and input radiance between 4.9 and 5.8 (relative sample reflectance between 0.34 and $0.4, c f$. fig. 7a) the radiance equivalent to noise in the data $\left(\mathrm{NER}_{\text {data }}\right)$ exceeds the sensor noise equivalent radiance $\left(\mathrm{NER}_{\text {sensor }}\right)$ by about $10 \%$. For radiance between 3.5 and $4.4 \mathrm{~W} * \mathrm{~m}^{-2} * \mu \mathrm{m}^{-1} * \mathrm{sr}^{-1}$ (sample reflectance between 0.24 and 0.3 ), $\mathrm{NER}_{\text {data }}$ exceeds the $\mathrm{NER}_{\text {sensor }}$ by up to $40 \%$. Sample no. 10 , with radiance above $7.5 \mathrm{~W} * \mathrm{~m}^{-2} * \mu \mathrm{m}^{-1} * \mathrm{sr}^{-1}$ and reflectance above 0.5 , represents an exception. 


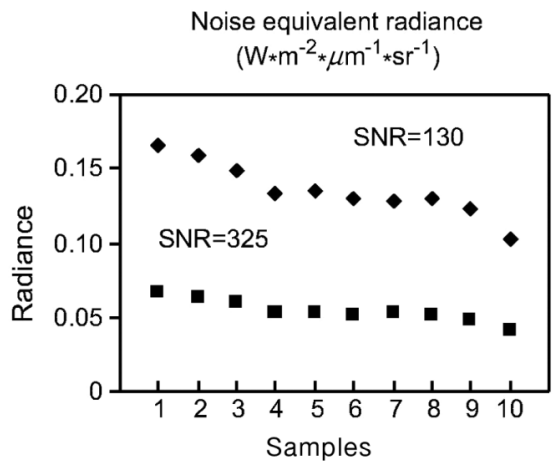

\begin{tabular}{|c|c|}
\hline \multicolumn{2}{|c|}{$\begin{array}{l}\text { At sensor radiance } \\
\left(\mathrm{W} * \mathrm{~m}^{-2} * \mu \mathrm{m}^{-1} * \mathrm{sr}^{-1}\right)\end{array}$} \\
\hline 1 & 3.545 \\
\hline 2 & 3.811 \\
\hline 3 & 4.382 \\
\hline 4 & 4.929 \\
\hline 5 & 5.089 \\
\hline 6 & 5.162 \\
\hline 7 & 5.147 \\
\hline 8 & 5.333 \\
\hline 9 & 5.827 \\
\hline 10 & 7.602 \\
\hline
\end{tabular}

For SNR 130: NER data $=0.102$ to $0.166 \mathrm{~W} * \mathrm{~m}^{-2} * \mu \mathrm{m}^{-1} * \mathrm{Sr}^{-1} ; 91 \%$ to $148 \%$ of $\mathrm{NER}_{\text {sensor }}$

For SNR 325: NER data $=0.041$ to $0.066 \mathrm{~W} * \mathrm{~m}^{-2} * \mu \mathrm{m}^{-1} * \mathrm{sr}^{-1}$

Fig. 8. Radiance equivalent to the noise in the data (NER data) for two sensor SNRs; the samples are ordered according to increasing reflectance. Sensor NER (NER sensor) is the mean, for all sensor channels, of the NER determined during the sensor calibration. See the text for explanation.
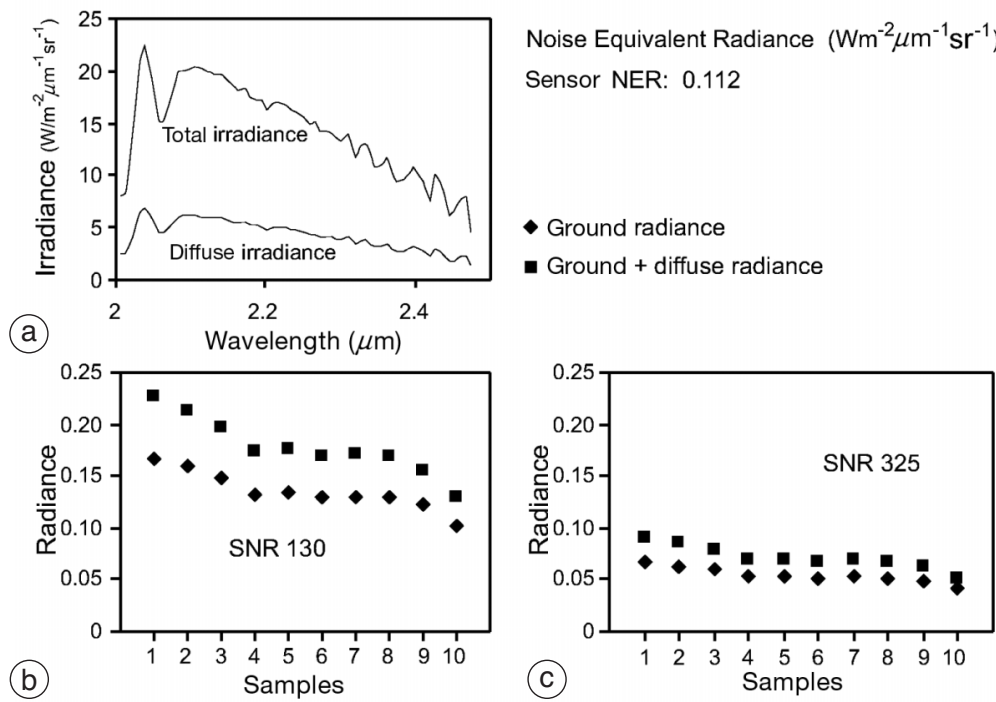

- Ground radiance

- Ground + diffuse radiance

(a)

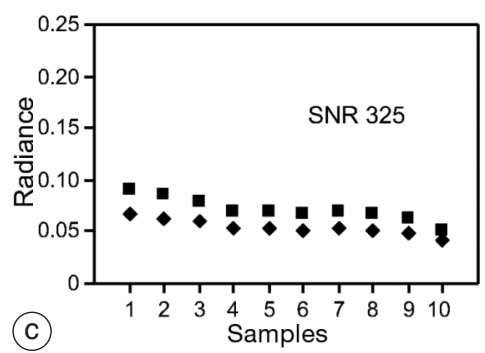

SNR 130

SNR 325

NER $R_{\text {data }}$ increments $\left(\mathrm{W} * \mathrm{~m}^{-2} * \mu \mathrm{m}^{-1} * \mathrm{sr}^{-1}\right)$

Min $=0.027$

NER $R_{\text {data }}$ increments $\left(\mathrm{W} * \mathrm{~m}^{-2} * \mu \mathrm{m}^{-1} * \mathrm{sr}^{-1}\right)$

$\operatorname{Min}=0.011$

Mean $=0.017$

Mean $=0.043$

$\operatorname{Max}=0.023$

Fig. 9a-c. a) Total and diffuse atmospheric irradiance measured in the field during a MIVIS overflight on the area of fig. 1a. Diffuse irradiance contributes to NER data up to about $190 \%$ of the NER sensor. $_{\text {b }}$ c) Increments of NER $_{\text {data }}$ (Noise Equivalent Radiance) calculated with total at sensor radiance, i.e. including the diffuse atmospheric component (squares) with respect to $\mathrm{NER}_{\text {data }}$ calculated without diffuse atmospheric component (triangles), for b) $\mathrm{SNR}=130$ and c) $\mathrm{SNR}=325$. See the text for discussion. 
For $\mathrm{SNR}=325$, the radiance increment due to noise would be reduced to less than one half.

All the topics discussed above and in the previous sections assume the absence of atmospheric scattering. Given an amount of diffuse radiance directly reaching the sensor, the noise in the data and the corresponding $\mathrm{NER}_{\text {data }}$ can be calculated

$$
\begin{aligned}
& S_{\text {noisy }}=\left\{\left[\left(\left(\left(S_{\text {lab }} * E_{\text {solar }}+\text { Diff }\right) * G+O\right) *\right.\right.\right. \\
& *(1+N(0,1) / \mathrm{SNR})-O) / G]- \text { Diff }\} / E_{\text {solar }}
\end{aligned}
$$

where Diff is the wavelength dependent diffuse radiance component. Figure 9a shows the total and diffuse solar irradiance measured in the field during a MIVIS flight in the area of fig. 1a. Diffuse irradiance interacts with the noise in the data, and as such, even when totally removed from output data during transformation to reflectance, it also affects the $\mathrm{NER}_{\text {data }}$, with significant increments compared to the $\mathrm{NER}_{\text {data }}$ without diffuse radiance. For a sensor $\mathrm{SNR}=130$ (fig. 9b), the $\mathrm{NER}_{\text {data }}$ with diffuse radiance component is from $27 \%$ to $37 \%$ higher than the $\mathrm{NER}_{\text {data }}$ without scattering, and it can be more than twice the sensor NER for low radiance samples. The NER increment due to scattering is almost negligible for a sensor with $\mathrm{SNR}=325$ (fig. 9c).

\section{Discussion and conclusions}

New generations of sensors for remote sensing have increasingly higher spectral and spatial resolutions, and consequently remote spectroscopy will be more and more comparable to laboratory spectroscopy. The advent of new imaging interferometer systems will probably overcome the intrinsic physical constraints of the optical dispersion sensors (e.g., Otten, 1997; Barducci et al., 2001).

Nevertheless, a few questions remain open. A first question is to what extent the measuring precision is congruent with the «determination degree» of the target, where this term expands the concept of detection limit for individual absorption features, taking into account the inherent variability of the geologic materials. In fact, high spectral resolution allows us to identify the processes associated with the mineralogical composition, as long as it reveals chemical and crystal structure characteristics. However, the rock-generating geologic processes, involving first order variables such as pressure, temperature and time, often result in continua between two or more endmember minerals or mineral associations. As discussed in this paper, some of these variations are systematically related to genetic processes, and in this case they can be modeled and, to some extent, predicted. Other variations, particularly those deriving from sedimentary and alteration processes, can be analyzed and, in some cases, even modeled, although rarely predictable. Although many studies have already been carried out to determine the spectral properties of geologic materials for both mineral identification and abundance determination, further work is needed in the fields of laboratory spectroscopy, petrographic and geochemical analyses of both natural and pure mineral synthetic mixtures, and radiation transfer theory for particulate materials.

A second question is to what extent the experimental conditions are under control, i.e. whether and to what extent the remotely sensed data are correlated with the target. As discussed in this paper, sensor radiometric calibration is of primary importance for noise minimization, the presence of which makes ambiguous any mineral identification and abundance quantification. No less important is the suppression of atmospheric effects. The several atmospheric models and inversion routines (MODTRAN, 6S, ATCORR, among others) can probably be improved to get more reliable results, but more important, as many atmospheric parameters as possible have to be accurately measured on the ground concomitantly with data acquisition, to provide the physical constraints for the correction procedure.

The future of high resolution remote sensing thus relies upon a close collaboration among engineering designers, spectroscopy physicists, atmosphere and radiometry physicists, and geologists.

\section{REFERENCES}

Barducci, A., P. Marcoionni, I. Pippi and M. Poggesi (2001): Simulation of the performance of a stationary 
imaging interferometer for high resolution monitoring of the Earth, in Sensors, Systems and Next Generation Satellites, edited by H. FuJISADA, SPIE Proc., 4540A.

BOARDMAN, J.W. (1993): Automated spectral unmixing of AVIRIS data using convex geometry concepts, in Summaries of the Fourth JPL Airborne Geoscience Workshop, Jet Propulsion Laboratory Publ. 93-26, 1, 11-14.

Clark, R.N. (1995): Reflectance spectra, in Rock physics and Phase Relations. A handbook of Physical Constant, Am. Geophys. Un. Ref. Shelf 3, 178-187.

Clark, R.N. and T.L. Roush (1984): Reflectance spectroscopy: quantitative analysis thechniques for remote sensing applications, J. Geophys. Res., 89, 6329-6340.

Crotti, M., I. Longhi, S. Meli and M. Sgavetti (2002): Dati spettroscopici e di laboratorio su sabbie litorali: le zone di foce adige e laguna Marano-Grado, Acta Nat. Ateneo Parmense, 38, 113-132.

DuKE, F.E. (1994): Near infrared spectra of muscovite, Tschermak substitution, and metamorphic reaction progress: Implications for remote sensing, Geology, 22, 621-624.

Longhi, I., C. Mazzoli and M. SgavetTi (2000): Determination of metamorphic grade in siliceous muscovitebearing rocks in Madagascar using reflectance spectroscopy, Terra Nova, 12, 21-27.

Longhi, I., M. Sgavetti, R. Chiari and C. Mazzoli (2001): Spectral analysis and classification of metamorphic rocks from laboratory reflectance spectra in the 0.4-2.5 $\mu \mathrm{m}$ interval: a tool for hyperspectral data interpretation, Int. J. Remote Sensing, 22, 3763-3782.

Longhi, I., M. Sgavetti, S. Meli and L. Pompilio (2004): Complex spectral interactions of different minerals and textures in Mars terrestrial analogues: some examples, Planet. Space Sci., 52, 141-147.
McMillan, P.F. and A.M. Hofmeister (1988): Infrared and Raman spectroscopy, in Spectroscopic Methods, edited by F.C. Howthorne, Rev. Mineral., 18, 99-160.

NASH, D.B. and J.E. Conel (1974): Spectral reflectance systematics for mixtures of powdered hypersthene, labradorite, and illmenite, J. Geophys. Res., 79, 16151621.

OKING, G.S. and D.A. RoBERTs (2000): Linear unmixing of simulated, noisy spectra: vegetation detection limitations in areas of low cover, in Proceedings of the AVIRIS Workshop (on line: http//makalu.jpl.nasa.gov).

OTTIN, L.J. (1997): LWIR and MWIR ultraspectral Fourier transform imager, SPIE Proc., 4331, 421-428.

Sgavetti, M., A. Gaibazzi, I. Longhi, S. Meli, C. GiardiNo, R. CAPElletti, A. BARAldi and M. CROTTI (2001): Accuracy of imaging spectroscopy for geologic material identification: analysis of noise effects, in Remote Sensing for Environmental Monitoring, GIS Applications, and Geology, edited by M. EHLERS, SPIE Proc., 4545, 184-195.

SingER, R.B. (1981): Near-infrared spectral reflectance of mineral mixtures: systematic combinations of pyroxenes, olivine, and iron oxides, J. Geophys. Res., 86, 7967-7982.

Singer, R.B. and T.B. McCord (1979): Mars: large scale mixing of bright and dark surface materials and implications for analysis of spectral reflectance, in Proceedings of the 10th Lunar and Planetary Science Conference, 1835-1848.

Swayze, G.A., R.N. Clark, F. Cruse, S. Sutley and A. GalLAGHER (1992): Ground-truthing AVIRIS mineral mapping at Cuprite, Nevada, in Summaries of the Third Annual JPL Airborne Geoscience Workshop, edited by R.O. GREEN, Jet Propulsion Laboratory Publ. 92-14, 1, 47-49. 